\title{
The role of neoadjuvant chemotherapy in patients with advanced (stage IIIC) epithelial ovarian cancer
}

\author{
Erik Škof ${ }^{1}$, Sebastjan Merlo², Gasper Pilko², Borut Kobal ${ }^{3}$ \\ ${ }^{1}$ Department of Medical Oncology, Institute of Oncology Ljubljana, Ljubljana, Slovenia \\ ${ }^{2}$ Department of Surgery, Institute of Oncology Ljubljana, Ljubljana, Slovenia \\ ${ }^{3}$ Division of Gynaecology and Obstetrics, University Medical Centre Ljubljana, Ljubljana, Slovenia
}

Radiol Oncol 2016; 50(3): 341-346.

Received 29 May 2015

Accepted 5 September 2015

Correspondence to: Borut Kobal, M.D., Division of Gynaecology and Obstetrics, University Medical Centre Ljubljana, Ljubljana, Zaloška 2, SI-1000 Ljubljana, Slovenia. E-mail: borut.kobal@kclj.si

Disclosure: The authors declare no conflict of interest.

Background. Primary treatment of patients with advanced epithelial ovarian cancer consists of chemotherapy either before (neoadjuvant chemotherapy, NACT) or after primary surgery (adjuvant chemotherapy). The goal of primary treatment is no residual disease after surgery (RO resection) what is associated with an improvement in survival of patients. There is, however, no evidence of survival benefits in patients with RO resections after prior NACT.

Methods. We retrospectively reviewed the records of patients who were treated with diagnosis of epithelial ovarian cancer at Institute of Oncology Ljubljana in the years 2005-2007. The differences in the rates of R0 resections, progression free survival (PFS), overall survival (OS) and in five-year and eight-year survival rates between patients treated with NACT and patients who had primary surgery were compared.

Results. Overall 160 patients had stage IIIC epithelial ovarian cancer. Eighty patients had NACT and eighty patients had primary surgery. Patients in NACT group had higher rates of RO resection ( $42 \%$ vs. $20 \% ; p=0.011)$ than patients after primary surgery. PFS was 14.1 months in NACT group and 17.7 months after primary surgery $(p=0.213)$. OS was 24.8 months in NACT group and 31.6 months after primary surgery $(p=0.012)$. In patients with RO resections five-year and eight-year survival rates were $20.6 \%$ and $17.6 \%$ in NACT group compared to $62.5 \%$ and $62.5 \%$ after primary surgery $(p<0.0001)$, respectively.

Conclusions. Despite higher rates of RO resections achieved by NACT, survival of patients treated with NACT was inferior to survival of patients who underwent primary surgery. NACT should only be offered to patients with advanced epithelial cancer who are not candidates for primary surgery.

Key words: advanced ovarian cancer; neoadjuvant chemotherapy; primary surgery

\section{Introduction}

The standard treatment of patients with advanced epithelial ovarian cancer is a combination of primary surgery followed by chemotherapy. In the recent years it became clear that the goal of surgery is to achieve no macroscopic residual disease, since the survival of patients with no residual disease is superior to survival of patients with visible residual disease. ${ }^{1}$ To achieve this goal, several aggressive surgical techniques have been proposed. Often multivisceral resections are performed (diaphragm resection, splenectomy, colon resection, extensive peritonectomy, etc. $)^{2}$, which increase morbidity of patients.

Not all patients are candidates for primary surgery, either due to extend of their disease (unlikely to achieve no residual disease) or due to poor general condition (too ill to undergo an extensive operation). In this situation patients are treated with initial (neoadjuvant) chemotherapy, typically a combination of a platinum-based drug and a tax- 
ane. ${ }^{3}$ Patients, who are treated with neoadjuvant chemotherapy, are more likely to undergo surgery with no residual disease than patients with primary surgery. ${ }^{4}$ Therefore many authors believe that neoadjuvant chemotherapy is justified in order to have best chance to achieve a status of no residual disease. ${ }^{4,5}$

However, treatment with neoadjuvant chemotherapy has not yet been shown to provide better survival than treatment with primary surgery. ${ }^{4,6}$ It has been shown that survival of patients with stage III or IV who have no residual disease after primary surgery can be up to $50 \%$ at 10 years. ${ }^{7}$ It is not known, if patients who achieve a status of no residual disease with neoadjuvant chemotherapy,

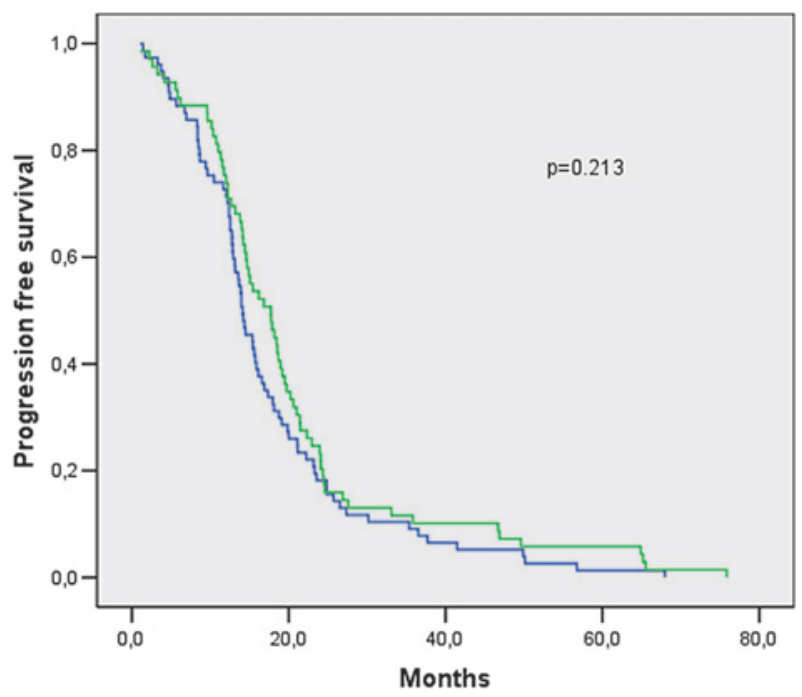

Primary surgery? 0-no, 1-yes $\bigcap_{0}$ $\neg 1$

FIGURE 1. Progression free survival of patients after primary surgery and neoadjuvant chemotherapy (NACT).

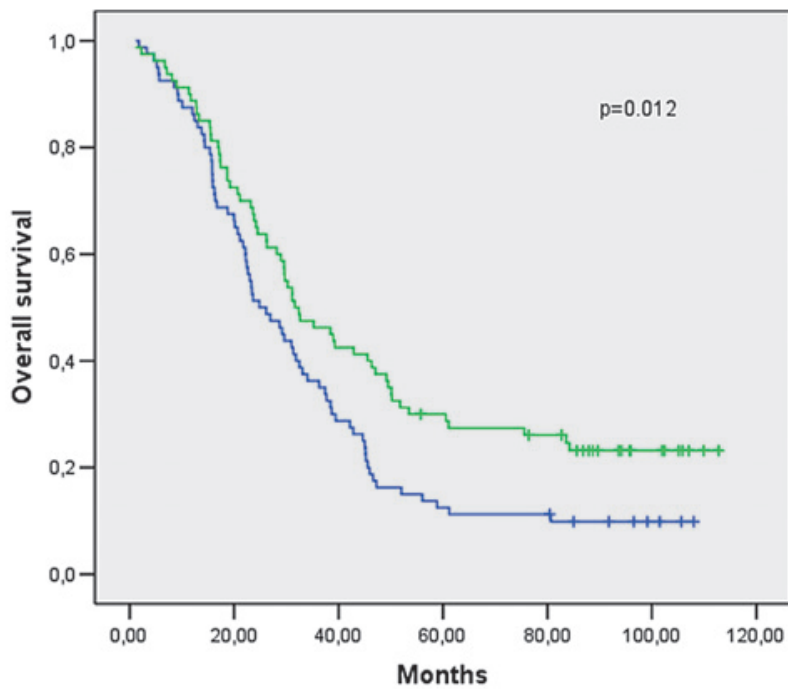

Primary surgery? 0 -no, 1-yes

$\bigcap_{0}$

$\Pi 1$

+0 -censored

+1 -censored

FIGURE 2. Overall survival of patients after primary surgery and neoadjuvant chemotherapy (NACT). have the same or equally good prognosis. There are data from one randomized trial which showed similar survival of patients treated with neoadjuvant chemotherapy to those treated with primary surgery, but, this study was criticized due to poor survival rates in both groups. ${ }^{4}$

We compared the differences in the rates of no residual disease (R0 resection) after surgery and the differences in five-year and eight-year survival rates in patients treated with neoadjuvant chemotherapy or primary surgery in correlation to the extent of residual disease post-surgery.

\section{Methods}

\section{Patients}

We retrospectively reviewed the records of patients who were treated with diagnosis of epithelial ovarian cancer at Institute of Oncology Ljubljana in the period from $1^{\text {st }}$ of January 2005 until $31^{\text {st }}$ of December 2007. During this period we identified 346 patients who were treated for epithelial ovarian cancer. Of these 160 patients had stage FIGO IIIC disease - they were eligible for analysis. Of the 160 eligible patients, 80 patients had neoadjuvant chemotherapy (NACT) and 80 patients had primary surgery.

Patients had primary surgery in seven different hospitals performed by many gynecologic surgeons. In all patients, hysterectomy, bilateral oophorectomy, infracolic omentectomy with limited peritonectomy was performed. In only few of the patients multivisceral resections (diaphragm resection, splenectomy, colon resection, extensive peritonectomy) were performed - therefore no comparison between different surgical techniques outcomes was carried out.

In the NACT group $40 \%$ of patients received platinum and taxane therapy (paclitaxel $175 \mathrm{mg}$ in 3h i.v. infusion and carboplatin AUC 6 i.v. infusion) and $55 \%$ of patients received carboplatin (AUC 6) monotherapy before surgery. Post-surgery all patients received 3 additional cycles of chemotherapy - the same regimen as in neoadjuvant setting. Median number of cycles in NACT group was 7 (range 1-13).

Of the 80 patients, who had primary surgery, $82 \%$ of them received platinum and taxane therapy (paclitaxel $175 \mathrm{mg}$ in $3 \mathrm{~h}$ i.v. infusion and carboplatin AUC 6 i.v. infusion), 16\% of patients received carboplatin (AUC 6) monotherapy. Median number of cycles of chemotherapy was 6 (range 1-9). All patients were treated with chemotherapy at 
Institute of Oncology Ljubljana by medical oncologist specialized for gynecologic oncology.

Reasons given for NACT included extent of disease $(50 \%)$, co-morbidities $(10 \%)$, and poor performance $(40 \%)$. For patients who had NACT, stage was established by combination of clinical examination, imaging (US, CT of abdomen), cytology and biopsy. For patients who were treated with primary surgery, stage was established by intraoperative examination and review pf pathology reports. The extent of residual disease was based on the diameter of the single largest lesion. Patients without macroscopic evident residual lesions had $\mathrm{R} 0$ resection, patients with less than $10 \mathrm{~mm}$ residual lesions had $\mathrm{R} 1$ resection, patients with residual lesions of $10 \mathrm{~mm}$ or more had $\mathrm{R} 2$ resection.

Our retrospective study was approved by the Institutional Review Board and Ethics Committee.

\section{Analysis}

We compared patients who had NACT with those who had primary surgery for a range of clinical variables. The extent of residual disease post-surgery was measured. We observed the differences in the rates of no residual disease after surgery, differences in progression free survival (PFS), overall survival (OS) and in five-year and eight-year survival rates in patients after various treatments. Patients were followed from the date of diagnosis until death from ovarian cancer, death from another cause or lost from follow-up.

The primary endpoints were OS, five-year and eight-year survival rates. The secondary end points were R0 resection rates and PFS.

Survival curves were calculated by KaplanMeier's method. Univariate and multivariate analyses using log-rank test and Cox's regression model were used for the assessment of the factors associated with OS and for comparison of factors between patients who had NACT with those who had primary surgery. Quantitative variables were compared using Student or the Wilcoxon test. Categorical variables were compared using Chisquare test. The differences were considered statistically significant if the $p$ values were less than 0.05 . Software package SPSS 15.0 for Windows was used.

\section{Results}

We analyzed 160 patients with stage IIIC epithelial ovarian cancer who were treated at Institute of Oncology Ljubljana in the period from $1^{\text {st }}$ of
TABLE 1. The characteristics of patients and surgical outcomes

\begin{tabular}{lccc}
\hline & $\begin{array}{c}\text { Primary surgery } \\
\mathbf{N}=80\end{array}$ & $\begin{array}{c}\text { NACT } \\
\mathbf{N}=80\end{array}$ & $\mathrm{p}$ \\
\hline Age (years) & 60.2 & 64.8 & 0.006 \\
$\begin{array}{l}\text { High grade serous } \\
\text { Performance } \\
\mathbf{0} \text { or } 1 \\
>1\end{array}$ & $43(54 \%)$ & $53(66 \%)$ & 0.307 \\
R0 resection & $66(83 \%)$ & $32(40 \%)$ & $<0.0001$ \\
R1 resection & $14(17 \%)$ & $48(60 \%)$ & 0.011 \\
R2 resection & $16(20 \%)$ & $34(42 \%)$ & 1.0 \\
Inoperable & $18(23 \%)$ & $18(22 \%)$ & $<0.0001$ \\
PFS (months) & $46(57 \%)$ & $11(14 \%)$ & - \\
OS (months) & - & $17(21 \%)$ & 0.213 \\
\hline
\end{tabular}

$\mathrm{NACT}$ = neoadjuvant chemotherapy; OS = overall survival; PFS = progression-free survival; $\mathrm{RO}$ = no macroscopic residual disease; $R 1=<1 \mathrm{~cm}$ residual disease; $R 2=>1 \mathrm{~cm}$ residual disease

TABLE 2. Five-year and eight-year survival of patients after different surgical outcomes

\begin{tabular}{lccc}
\hline & Five-year survival & Eight year survival & p \\
\hline Surgery - R0 resection & $62.5 \%$ & $62.5 \%$ & $\mathrm{P}<0.0001$ \\
NACT - R0 resection & $20.6 \%$ & $17.1 \%$ & \\
Surgery - R1 resection & $38.9 \%$ & $27.8 \%$ & $\mathrm{P}<0.0001$ \\
NACT - R1 resection & $16.7 \%$ & $11.1 \%$ & \\
Surgery - R2 resection & $15.5 \%$ & $0 \%$ & $\mathrm{P}<0.0001$ \\
NACT - R2 resection & $0 \%$ & $0 \%$ & - \\
Inoperable disease & $0 \%$ & $0 \%$ & - \\
\hline
\end{tabular}

$\mathrm{NACT}=$ neoadjuvant chemotherapy; $\mathrm{RO}=$ no macroscopic residual disease; $\mathrm{R} l=<1 \mathrm{~cm}$ residual disease; $\mathrm{R} 2=>1 \mathrm{~cm}$ residual disease

January 2005 until $31^{\text {st }}$ of December 2007. Median follow-up of patients was 8.4 years (range 6.7-10 years). Of 160 patients, 80 patients had primary surgery and 80 patients had NACT.

The characteristics of patients and surgical outcomes of patients who had primary surgery or NACT are shown in Table 1. Patients treated with primary surgery were younger (60.2 vs. 64.8 years; $\mathrm{p}<0.001)$. Patients treated with primary surgery also had a better performance status according to WHO classification ( $\mathrm{p}<0.001)$.

In patients treated with NACT higher rates of R0 resection were observed ( $42 \%$ vs. $20 \%$; p < 0.001 ) and lower rates of $\mathrm{R} 2$ resection rates (14\% vs. $57 \%$; $\mathrm{p}<0.001)$ compared to patients who had primary surgery. After NACT in $21 \%$ of patients disease remained to be inoperable.

Median PFS of patients was 14.1 months after NACT and 17.7 months after primary surgery (Figure 1). The difference was not statistically sig- 
TABLE 3. Factors correlated with survival on univariate analysis

\begin{tabular}{|c|c|}
\hline & $p$ \\
\hline $\begin{array}{r}\text { Age (years) } \\
\qquad 60 \\
\geq 60\end{array}$ & 0.008 \\
\hline $\begin{array}{l}\text { Histology } \\
\text { High grade serous } \\
\text { Other histology }\end{array}$ & 0.066 \\
\hline $\begin{array}{c}\text { Performance status } \\
0 \text { or } 1 \\
>1\end{array}$ & $<0.0001$ \\
\hline $\begin{array}{l}\text { Extent of residual disease } \\
\text { R0 } \\
\text { R1 } \\
\text { R2 }\end{array}$ & $<0.0001$ \\
\hline $\begin{array}{l}\text { Therapy } \\
\text { Primary surgery } \\
\text { NACT }\end{array}$ & 0.012 \\
\hline
\end{tabular}

NACT = neoadjuvant chemotherapy

TABLE 4. Factors correlated with survival on multivariate analysis

\begin{tabular}{|c|c|}
\hline & $p$ \\
\hline $\begin{array}{l}\text { Age (years) } \\
\begin{aligned} & <60 \\
& \geq 60\end{aligned}\end{array}$ & 0.775 \\
\hline $\begin{array}{l}\text { Histology } \\
\text { High grade serous } \\
\text { Other histology }\end{array}$ & 0.370 \\
\hline $\begin{array}{l}\text { Performance status } \\
\qquad \begin{array}{c}0 \text { or } 1 \\
>1\end{array}\end{array}$ & 0.003 \\
\hline $\begin{array}{l}\text { Extent of residual disease } \\
\text { R0 } \\
\text { R1 } \\
\text { R2 }\end{array}$ & $<0.0001$ \\
\hline $\begin{array}{l}\text { Therapy } \\
\text { Primary surgery } \\
\text { NACT }\end{array}$ & 0.038 \\
\hline
\end{tabular}

$\mathrm{NACT}=$ neoadjuvant chemotherapy

nificant $(\mathrm{p}=0.213)$. Median OS of patients was 24.8 months after NACT and 31.6 months after primary surgery (Figure 2). The difference was statistically significant $(p=0.012)$.

Five-year and eight-year survival rates of patients after different surgical outcomes are shown in Table 2. Patients treated with primary surgery had superior five-year and eight-year survival rates in all types of surgical outcomes compared to patients treated with NACT. Five-year and eight year survival rates were $62.5 \%$ and $62.5 \%$ vs. $20.6 \%$ and $17.1 \%$ after $\mathrm{R} 0$ resection ( $\mathrm{p}<0.001), 38.9 \%$ and $27.8 \%$ vs. $16.7 \%$ and $11.1 \%$ after $\mathrm{R} 1$ resection ( $\mathrm{p}<$ 0.001 ) and $15.5 \%$ and $0 \%$ vs. $0 \%$ and $0 \%$ after R2 resection $(\mathrm{p}<0.0001)$, respectively. Survival curves of patients after different surgical outcomes are shown in Figure 3.

Univariate analysis showed that factors associated with survival were: performance status according to WHO ( $<<0.0001)$, extent of residual disease $(p<0.0001)$, primary surgery $(p=0.012)$ and age $(\mathrm{p}=0.008)$ (Table 3$)$.

We conducted multivariate survival analysis using Cox's regression model. The following prognostic factors in the multivariable model: patient age at diagnosis ( $<60$ vs. $\geq 60$ years); extent of residual disease (R0, R1, R2); chemotherapy (NACT vs. primary surgery); performance status according to $\mathrm{WHO}(0$ or 1 vs. > 1) and histopathological subtype (high grade serous vs. other) were entered. Patients treated with primary surgery had better survival also on multivariate survival analysis. Besides that, other independent predictors of survival were extent of residual disease and performance status (Table 4).

\section{Discussion}

Our results show that treatment with NACT doubles the chance to have no visible residual disease (R0 resection) post-surgery compared to primary surgery in patients with stage IIIC epithelial ovarian cancer $(42 \%$ vs. $20 \%)$. This is in concordance with already published studies. ${ }^{4,5}$ Despite higher rates of R0 resections achieved by NACT, survival of patients treated with NACT was inferior to survival of patients who underwent primary surgery by almost 7 months (24.8 months vs. 31.6 months). Even in patients who had $\mathrm{R} 0$ resection post-surgery, there was a huge difference in probability to be fiveyear or eight-year survivor in favor of primary surgery. Patients with R0 resection at primary surgery had three-fold higher rates of five-year and eightyear survival rates than patients with R0 resection after NACT (62.5\% vs. $20.6 \%$ and $62.5 \%$ vs. $17.1 \%$, respectively). Our results are in concordance with many authors who reported similar results with inferior overall survival of patients after NACT despite higher rates of optimal debulking surgery. ${ }^{5-8}$

There have been published results of a randomized trial which showed similar survival of patients treated with NACT to those treated with primary surgery, but, this study was criticized due to poor survival rates in both groups. ${ }^{4}$

The limitation of our study was, that this was not a randomized trial, therefore at least to some extent the difference in the outcomes for the different groups might be the result of an imbalance in the baseline characteristics. Patients who received 
NACT were more likely to have more extensive disease at diagnosis than patients who had primary surgery, and therefore it was expected to do relatively worse. Patients who received NACT were almost five years older (64.8 years vs. 60.2 years), had more co-morbidities and had worse performance than patients who had primary surgery. Half of the patients who received NACT did not receive combination of taxane and carboplatin chemotherapy due to poor performance or co-morbidities, whereas vast majority of patients received combination of taxane and carboplatin after primary surgery.

Multivariate analysis showed that independent predictors of survival were primary surgery, performance status and extent of residual disease. Therefore, a profound differences in survival among patients after R0 resection (and also R1 resection) between primary surgery and NACT can be at least to some extend explained by factors, other than disease itself.

Authors believe that it is improper to compare survival of patients who had $\mathrm{R} 0$ resection at primary surgery to patients who had $\mathrm{R} 0$ resection after prior NACT if the patients are not balanced regarding performance status, age, comorbidities, etc. It is like comparing apples with oranges.

It is not just the extent of the disease at diagnosis that is important for prognosis. The biology of the disease may also play an important role. At the moment there are no biologic markers that would help us to choose the best treatment strategy for patients with advanced epithelial ovarian cancer. It is known that the most common histologic type of epithelial ovarian cancer, high grade serous adenocarcinoma, is sensitive to chemotherapy, while other histologic types such as clear cell and mucinous ovarian cancer are resistant to chemotherapy. In the last years much effort has been done in discovering of predictive and prognostic molecular markers with molecular profiling of epithelial ovarian cancer. Recently published data have shown that at least three molecular subtypes of high-grade serous ovarian cancer exist, which may have different predictive and prognostic values in systemic treatment of patients with advanced epithelial ovarian cancer. ${ }^{9}$

At the moment there is still no consensus regarding the use of NACT. The lack of consensus on who are candidates for NACT was reflected at the fourth Gynecologic Cancer InterGroup (GCIG) consensus conference. ${ }^{10}$ While the majority of attendees felt that NACT was a standard option for all patients with advanced epithelial ovarian cancer, others felt that NACT should be offered to a more clearly defined subgroup of women in whom

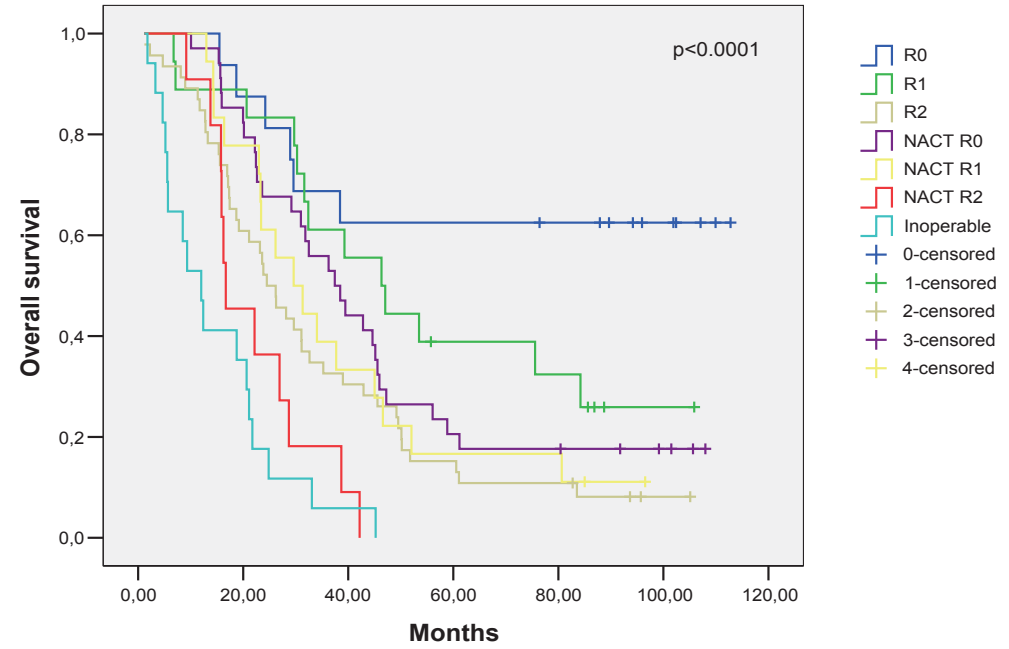

FIGURE 3. Survival curves of patients after different surgical outcomes.

$\mathrm{NACT}=$ neoadjuvant chemotherapy; $\mathrm{RO}=$ no macroscopic residual disease; $\mathrm{R} 1=<1 \mathrm{~cm}$ residual disease; $R 2=>1 \mathrm{~cm}$ residual disease

upfront surgery is contraindicated. The decision on whether to treat with NACT is based on the clinical status of the patient and whether or not disease is resectable at the time of presentation. Therefore, all patients require clinical staging. There are widely accepted criteria for unresectability. ${ }^{11}$ It is often difficult to preoperatively assess whether patients with advanced epithelial ovarian cancer can be optimally cytoreduced at the time of primary surgery. ${ }^{12}$ Therefore, many authors perform a staged surgical assessment for these patients and perform a diagnostic laparoscopy to further evaluate for resectability. If the surgeon conducting the assessment feels disease is resectable, primary surgery should be performed. If complete resectability is unlikely, NACT can be administered.

Despite better overall survival of our patients who had primary surgery, two-thirds of patients in this group had $\mathrm{R} 2$ resection $(>1 \mathrm{~cm}$ residual disease) which is considered as a sub-optimal resection. These patients had five-year survival rates of only $15.5 \%$ with none being alive at eight years post-surgery. In NACT group there were only 14 $\%$ of patients with $\mathrm{R} 2$ resection. We believe that the main reason for high rate of $\mathrm{R} 2$ resections at primary surgery was that primary surgery was performed in different hospitals mainly by surgeons not skilled with principles of oncology surgery, whereas all patients who had NACT underwent surgery provided by surgeon experienced in oncology. There are convincing data showing that surgical expertise plays a major role in outcome of patients with advanced epithelial ovarian cancer. In 
multidisciplinary cancer centers with advanced expertise in gynecologic oncology, optimal debulking rates in excess of $70 \%$ have been reported even for patients with bulky stage IIIC disease. ${ }^{7}$ Therefore, it is strongly recommended that a gynecologic oncologist must be involved in surgical decision making and treatment in these circumstances.

We believe there is enough evidence that in operable disease primary surgery with aim of no residual disease should be performed, since this offers best possible survival of patients with stage IIIC epithelial ovarian cancer. ${ }^{1}$ Of course patients must be fit enough for surgery, in which often multivisceral resections are needed with intention to achieve no residual disease post-surgery, which increase morbidity of patients. ${ }^{2}$ The NACT should only be offered to patients who are not candidates for primary surgery for whatever reason (advanced disease, poor performance, comorbidities, etc.). The aim of NACT should be to convert inoperable advanced disease to operable disease with goal to achieve optimal debulking (R0 resection). We believe that in patients with advanced epithelial ovarian cancer the aim of treatment should not only be the survival benefit but also the improvement in quality of life. Latter can be achieved by direct effect of chemotherapy on downsizing tumor burden, which offers higher chance for improvement in performance by lessening of the disease symptoms, offers higher chance to achieve optimal debulking at surgery, with less postoperative complications. ${ }^{4,5,13}$ Since this was a retrospective study, a comparison of the quality of life between patients treated with NACT or primary surgery was not performed.

One of the remarks towards NACT was that patients who started their treatment with NACT never had the chance for complete or optimal cytoreductive surgery. Since $48 \%$ of patients who received NACT in our study population were not candidates for radical or ultraradical surgical procedures as a result of poor performance status or comorbidities, they should probably be excluded from comparison with patients who were capable to undergo primary surgery. Perhaps the survival of patients who had NACT, but were otherwise fit for primary surgery, should be compared to survival of patients who had suboptimal primary surgery (R2 resection), thus comparing different treatments for similar burden of the disease. If we compare these two groups of patients we can see that five-years survival rates in NACT group with R0 or R1 post-surgery (NACT-R0 and NACT-R1) were somewhat higher compared to patients who had R2 resection at primary surgery $(20.6 \%$ after
NACT-R0 surgery, $16.7 \%$ after NACT-R1 surgery and $15.5 \%$ after $\mathrm{R} 2$ primary surgery), the difference was not statistically significant.

To conclude, our results show that treatment with NACT doubles the chance to have no visible residual disease (R0 resection) post-surgery compared to primary surgery in patients with advanced (stage IIIC) epithelial ovarian cancer. Despite higher rates of $\mathrm{R} 0$ resections achieved by NACT, survival of patients treated with NACT was inferior to survival of patients who underwent primary surgery by almost 7 months. Therefore we strongly believe that NACT should only be offered to patients with advanced epithelial cancer who are not candidates for primary cytoreductive surgery, or when the disease is unresectable after staged surgical assessment performed by oncology surgeon.

\section{References}

1. Aletti GD, Dowdy SC, Gostout BS, Jones MB, Stanhope CR, Wilson TO, et al. Aggressive surgical effort and improved survival in advanced-stage ovarian cancer. Obstet Gynecol 2006; 107: 77-85.

2. Eisenkop SM, Spirtos NM. Procedures required to accomplish complete cytoreduction of ovarian cancer: is there a correlation with "biological aggressiveness" and survival? Gynecol Oncol 2001; 82: 435-41.

3. Ozols RF, Bundy BN, Greer BE, Fowler JM, Clarke-Pearson D, Burger RA, et al. Phase III trial of carboplatin and paclitaxel compared with cisplatin and paclitaxel in patients with optimally resected stage III ovarian cancer: a Gynecologic Oncology Group study. J Clin Oncol 2003; 21: 3194-200.

4. Vergote I, Tropé CG, Amant F, Kristensen GB, Ehlen T, Johnson N, et al. Neoadjuvant chemotherapy or primary surgery in stage IIIC or IV ovarian cancer. N Engl J Med 2010; 363: 943-53.

5. Vergote I, Leunen K, Amant F. Primary surgery or neoadjuvant chemotherapy in ovarian cancer: what is the value of comparing apples with oranges? Gynecol Oncol 2012; 124: 1-2.

6. Bristow RE, Chi DS. Platinum-based neoadjuvant chemotherapy and interval surgical cytoreduction for advanced ovarian cancer: a meta-analysis. Gynecol Oncol 2006; 103: 1070-6.

7. Chi DS, Musa F, Dao F, Zivanovic O, Sonoda Y, Leitao MM, et al. An analysis of patients with bulky advanced stage ovarian, tubal, and peritoneal carcinoma treated with primary debulking surgery (PDS) during an identical time period as the randomized EORTC-NCIC trial of PDS vs neoadjuvant chemotherapy (NACT). Gynecol Oncol 2012; 124: 10-4.

8. Rosen B, Laframboise S, Ferguson S, Dodge J, Bernardini M, Murphy J, et al. The impacts of neoadjuvant chemotherapy and of debulking surgery on survival from advanced ovarian cancer. Gynecol Oncol 2014; 134: 462-7.

9. GourleyC, McCavigan A, Perren T, Paul J, OgilvieMichie C, Churchman M, et al. Molecular subgroup of high-grade serous ovarian cancer (HGSOC) as a predictor of outcome following bevacizumab. [Abstract]. J Clin Oncol 2014; 32(5 Suppl): abstract No. 5502

10. Vergote I, du Bois A, Amant F, Heitz F, Leunen K, Harter P. Neoadjuvant chemotherapy in advanced ovarian cancer: On what do we agree and disagree? Gynecol Oncol 2013; 128: 6-11.

11. Stuart GC, Kitchener H, Bacon M, duBois A, Friedlander M, Ledermann J, et al. 2010 Gynecologic Cancer InterGroup (GCIG) consensus statement on clinical trials in ovarian cancer: report from the Fourth Ovarian Cancer Consensus Conference. Int J Gynecol Cancer 2011; 21: 750-5.

12. Salani R, Bristow RE. Surgical management of epithelial ovarian cancer. Clin Obstet Gynecol 2012; 55: 75-95.

13. Thrall MM, Gray HJ, Symons RG, Weiss NS, Flum DR, Goff BA. Neoadjuvant chemotherapy in the Medicare cohort with advanced ovarian cancer. Gynecol Oncol 2011; 123: 461-6. 